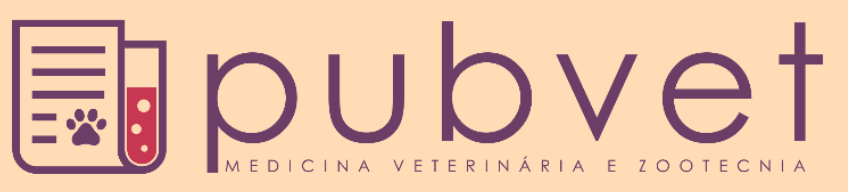

https://doi.org/10.31533/pubvet.v15n03a777.1-5

\title{
Análisis del efecto de diferentes dosis de enraizante en la productividad de soja vía modelo de regresión
}

\author{
Guido Gustavo Humada Gonzalez ${ }^{1}$, , Karina Franco Nuñez $^{2}$, Flavio Cavalheiro Zandonai ${ }^{2}$ \\ 'Profesor Doctor. Universidad San Carlos, Departamento de Producción Agrícola. Asunción - Paraguay. \\ ${ }^{2}$ Estudiante. Universidad San Carlos. Asunción - Paraguay. \\ ${ }^{2}$ Estudiante. Universidad San Carlos. Ciudad del Este - Paraguay. \\ *Autor para correspondencia, E-mail: gustavohumad@hotmail.com
}

Resumen. Con el objetivo de analizar el efecto de diferentes dosis de enraizante en cultivo de soja (Glycine max L. Merril) fue realizado un estudio basado en un experimento montado el Departamento de Caaguazú, con coordenadas $25^{\circ} 24^{\prime} 7.5^{\prime}$ 'S y $54^{\circ} 51^{\prime}$ '29.3'”O. El experimento se llevó a cabo en los meses de septiembre del 2018 a enero de 2019. Fueron evaluadas 20 unidades experimentales dispuestas en bloques completos al azar. Los tratamientos consistieron en cuatro diferentes dosis de enraizante $(0,2,4,6)$ expresados en $\mathrm{ml}$ con cinco repeticiones para cada uno de los tratamientos. Para establecer la dosis óptima de enraizante fue realizado un análisis de regresión utilizando el modelo lineal segmentado con respuesta plato. En las condiciones en que fue realizado el experimento los resultados muestran que la dosis óptima de enraizante Fertiactyl LEG que maximiza la productividad es igual a $5,08 \mathrm{ml} / \mathrm{kg}$ lo que permite obtener una productividad de $3.809 \mathrm{~kg} / \mathrm{há}$. El coeficiente de determinación demuestra además que el modelo lineal segmentado con respuesta plato, es adecuado para modelar eventos que relacionan dosis con rendimiento.

Palabras clave: Enraizante, modelo lineal segmentado con respuesta plato, rendimiento

\section{Analysis of the effect of different doses of rooting in soybean cultivation via regression model}

\begin{abstract}
To analyze the effect of different doses of rooting in soybean (Glycine max L. Merril) cultivation, an experiment was carried out in Caaguazu Departament with coordinates $25^{\circ} 24^{\prime} 7.5^{\prime}$ 'S y $54^{\circ} 51^{\prime} 29.3^{\prime \prime} \mathrm{W}$. The experiment was carried out between September 2018 and January 2019. Twenty experimental units were evaluated arranged in complete random blocks. The treatments consisted of four different kinds of rooting $(0,2,4,6)$ expressed in $\mathrm{ml}$ with 5 repetitions for each of the treatments. To establish the optimal dose of rooting, a regression analysis was performed using the segmented linear model with plate response. Under the conditions in which the experiments were carried out, the results show that the optimal dose of rooting which maximizes productivity is equal to $5,08 \mathrm{ml} / \mathrm{kg}$ of Fertiactyl LEG, which obtains a productivity of $3,809 \mathrm{~kg} / \mathrm{ha}$. The determination coefficient also proves that the segmented linear model with plate reply, is suitable to show events that are related to the yield vs doses.
\end{abstract}

Keywords: rootings, segmented linear model with plate reply, productivity 


\title{
Análises do efeito de diferentes doses de enraizante na produtividade de soja via modelo de regressão
}

\begin{abstract}
Resumo. Para analisar o efeito de diferentes doses de enraizamento no cultivo da soja
(Glycine max L. Merril) foi realizado um estudo a partir de um experimento instalado no Departamento de Caaguazú, com as coordenadas $25^{\circ} 24^{\prime} 7.5^{\prime}$ ' $\mathrm{S}$ e $54^{\circ} 51^{\prime}$ '29.3' O. O experimento foi realizado nos meses de setembro de 2018 a janeiro de 2019. Foram avaliadas 20 unidades experimentais dispostas em blocos ao acaso completos. Os tratamentos consistiram em quatro diferentes doses de agente de enraizamento $(0,2,4,6)$ expressas em $\mathrm{ml}$ com cinco repetições para cada um dos tratamentos. Para estabelecer a dose ideal de agente de enraizamento, foi realizada uma análise de regressão usando o modelo linear segmentado com uma resposta em placa. Nas condições em que o experimento foi realizado, os resultados mostram que a dose ótima do agente de enraizamento Fertiactyl LEG que maximiza a produtividade é igual a 5,08 $\mathrm{ml} / \mathrm{kg}$, o que permite obter uma produtividade de $3.809 \mathrm{~kg} / \mathrm{ha}$. O coeficiente de determinação também mostra que o modelo linear segmentado com uma resposta de placa é adequado para modelar eventos que relacionam a dose ao desempenho.
\end{abstract}

Palavras chave: Enraizamento, modelo linear segmentado com resposta de placa, rendimento

\section{Introducción}

Según PNCS (2016), la soja, constituye el principal cultivo agrícola de importancia económica que se produce en Paraguay. El país, a pesar de las limitaciones en cuanto a área disponible para siembra, se ubica entre los cinco mayores productores de la oleaginosa; además es el cuarto mayor exportador de soja a nivel mundial. Las semillas de soja se forman dentro de vainas y es considerada una planta anual que se cultiva en estaciones cálidas.

La extracción de elementos fertilizantes del suelo de una cosecha de soja es elevada, siendo el nitrógeno el elemento más demandado por el cultivo, pero, si se emplean inoculantes, la soja es capaz de maximizar el uso del nitrógeno obtenido del aire, con lo que la cantidad de este elemento, a añadir en forma mineral, se puede reducir de un modo notable (Hermoso, 1974).

Según Veronesi (2014), el estimulante se aplica en la semilla, con la ventaja de poder agregarlo a la inoculación, y debe ser aplicado momentos ante de la siembra. Entre las ventajas del uso de enraizantes se destaca la germinación rápida y uniforme, favoreciendo para una mejor nodulación y fijación del nitrógeno atmosférico, mayor desarrollo radicular y mayor capacidad de absorción de agua y nutrientes, con un elevado número de nódulos y más eficientes en cuanto a capacidad de fijación.

Con el objetivo de evaluar el efecto del estimulante Fertiactyl LEG en el rendimiento fueron analizados los datos provenientes de un experimento con el cultivo de soja, y determinar así, vía modelo de regresión segmentado con respuesta plato, la dosis que maximiza el rendimiento del cultivo.

\section{Materiales y métodos}

Los datos analizados corresponden a un experimento con soja (Glycine max L. Merril), variedad Nidera NA5909, montado en la segunda quincena de setiembre del 2018 en el departamento de Caaguazú, con coordenadas $25^{\circ} 24^{\prime} 7.5^{\prime}$ 'S y $54^{\circ} 51^{\prime} 29.3$ ' O. El suelo del lugar es clasificado como Rhodic kandiudox de orden Oxisol, de textura franco arcillosa y de origen basáltica con relieve plano a casi plano con menos de 3\% de pendiente (López et al., 1995). En la zona la temperatura media anual es de $25^{\circ} \mathrm{C}$ y durante el experimento se registraron $468 \mathrm{~mm}$ de lluvia.

Se utilizaron parcelas de $3 \mathrm{~m}$ de ancho por $4 \mathrm{~m}$ de largo cada una, con espaciamiento de $1 \mathrm{~m}$ entre tratamientos y bloques, totalizando una superficie de $470 \mathrm{~m}^{2}$, distribuidos en 20 unidades experimentales. La densidad de siembra utilizada para cada parcela experimental fue de 12 semillas por metro lineal, espaciadas $45 \mathrm{~cm}$ entre líneas de plantas. 
El diseño experimental utilizado fue, bloques completos al azar con 4 (cuatro) tratamientos y 5 (Cinco) repeticiones, donde fue analizada la variable rendimiento en kilogramos por hectárea. Los tratamientos utilizados en la investigación están representados en la tabla 1.

Tabla 1. Cantidad de enraizante (en mililitros) aplicados en experimento con soja

\begin{tabular}{lc}
\hline Tratamientos & Dosis de enraizante \\
\hline Tratamiento: 1 & Testigo (sin aplicación) \\
Tratamiento: 2 & $2 \mathrm{ml}$ \\
Tratamiento: 3 & $4 \mathrm{ml}$ \\
Tratamiento: 4 & $6 \mathrm{ml}$ \\
\hline
\end{tabular}

La composición del enraizante es: Complejo Fertiactyl + 0.5\% Cobalto + 3\% Molibdeno

Primeramente, antes de realizar la siembra, las semillas fueron tratadas con fungicidas a dosis de 1 $\mathrm{ml} / \mathrm{kg}$ de semilla, luego se le aplicaron insecticidas Thiamethoxam a dosis de $1 \mathrm{ml} / 100 \mathrm{Kg}$ de semilla y finalmente fueron inoculadas con enraizador Fertiactyl LEG, según la dosis estipulada para cada tratamiento (Tabla 1). Todos estos procedimientos se realizaron en el mismo día de la siembra. La siembra se realizó en forma mecánica con una sembradora manual de una línea. El control de plagas, enfermedades y malezas se realizó en forma manual conforme al requerimiento e incidencias en el cultivo. La cosecha de cada unidad experimental se realizó manualmente, considerando las hileras centrales de la parcela útil ( $2 \mathrm{~m}$ x 1,80 m), desechando los bordes y las cabeceras; dicho procedimiento fue efectuado cuando el cultivo alcanzo su madurez fisiológica. Posteriormente y antes de la trilla, se expuso las vainas al sol cuatro a cinco horas a los efectos de facilitar la apertura de las vainas y el secado de las semillas. Finalmente las semillas de las vainas de las plantas cosechadas y trilladas en la parcela útil fueron pesadas en una balanza electrónica de precisión y los valores resultantes (expresados en $\mathrm{kg} / \mathrm{ha}$ ) fueron registrados en una planilla. Tal operación se repitió para cada tratamiento y en cada una de sus respectivas repeticiones.

\section{Modelo de análisis de los datos e interpretación}

Para la estimación de la dosis optima del enraizante fue utilizado el modelo lineal segmentado con respuesta plato (MLSRP) descripto a continuación.

Según Braga (1983), el MLSRP consta de dos segmentos. El primero describe una recta creciente hasta una determinada constante $\mathrm{P}$, que es el plato, $\mathrm{y}$ el segundo segmento se refiere al plato, que a partir de un determinado valor del coeficiente de variación (CV) asume un valor constante. El modelo es representado por:

$$
y_{(i)}\left\{\begin{array}{rrr}
\beta_{0}+\beta_{1} X+\varepsilon_{i} & \text { si } & X_{i} \leq X_{0} \\
P+\varepsilon_{i} & \text { si } & X_{i}>X_{0}
\end{array}\right\}
$$

En que $y_{i}$ representa la respuesta (productividad media en kg.) al enraizante en dosis $X_{i}, X_{0}$ es el punto de unión entre los dos segmentos y determina la dosis óptima del enraizante, $P$ es la productividad del cultivo en el punto correspondiente al plato, $\beta_{0}$ y $\beta_{1}$ son los parámetros del segmento lineal y $\varepsilon_{i}$ es el error asociado al $y_{(i)}$ asumiendo normalidad e independientemente distribuido con media $0 \mathrm{y}$ variancia $\sigma_{\varepsilon}^{2}$ constante.

Los dos segmentos, lineal y plato, son igualados en el punto de $X_{0}$. Para obtener $X_{0}$ se toma $\beta_{0}+$ $\beta_{1} X_{0}=P$. Despejando $X_{0}$ de $\beta_{0}+\beta_{1} X_{0}=P$ se tiene la expresión que determina la dosis óptima que es dada por

$$
X_{0}=\left(P-\beta_{0}\right) / \beta_{1}
$$

En que $X_{0}$ representa la dosis optima del enraizante.

Para la evaluación de la calidad de ajuste del modelo fue calculado el coeficiente de determinación $\left(\mathrm{R}^{2}\right)$. Todos los cálculos necesarios para llegar a las conclusiones fueron realizados utilizando el programa R (R Developmen Core Team, 2020). 


\section{Resultados y discusión}

En el grafico 1, se observa la relación entre la productividad del cultivo de la soja y la dosis de enraizante colocado en las semillas momentos antes de la siembra. Se puede observar que el comportamiento productivo de la soja amplia con el aumento de la dosis, lo cual se ve representado claramente en el primer segmento del modelo de regresión. Luego, llega a un punto donde se estabiliza la productividad (punto óptimo o dosis óptima). A partir de ese punto el incremento de dosis del enraizante ya no tiene efecto sobre la productividad y es representado por el segundo segmento del modelo.

Soja cultivar Nidera NA5909

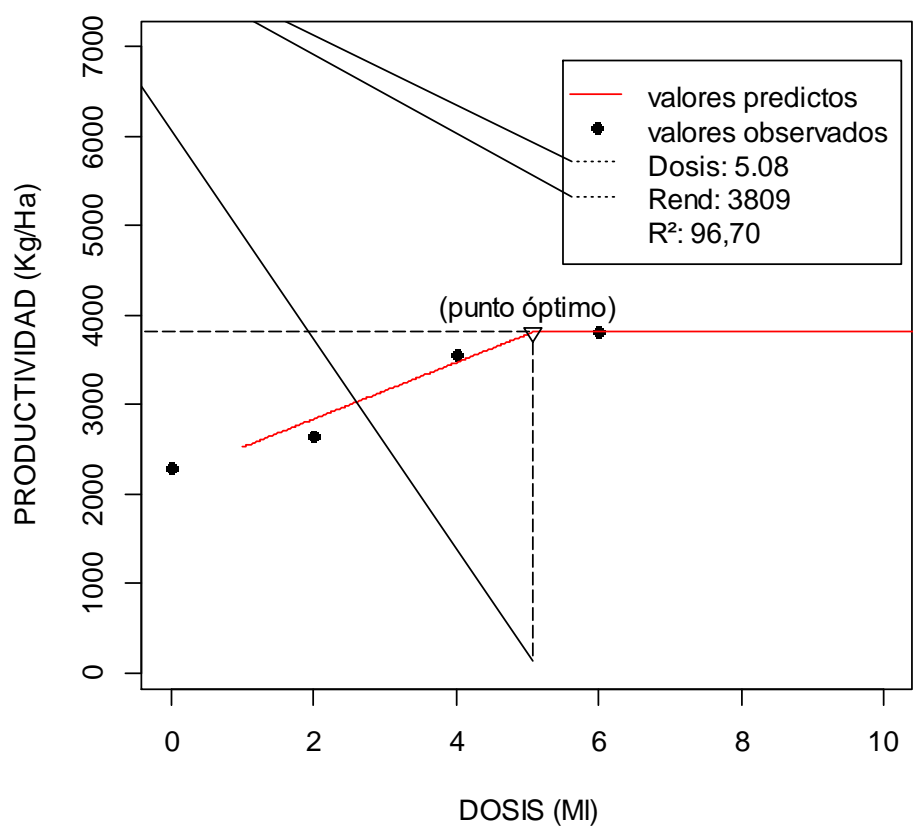

Gráfico 1. Representación gráfica del comportamiento de la productividad de la soja en función a la cantidad de enraizante adicionado

Delante de lo expuesto, la dosis óptima estimada, via modelo lineal segmentado con respuesta plato, para maximizar la productividad del cultivo es de 5,08ml por kilogramo de semilla de soja. Este resultado difiere de lo encontrado por Cavalheiro (2019), que en un trabajo de evaluación del efecto de las dosis de enraizante en el cultivo de la soja, determino que la dosis que maximiza la productividad del cultivo $(3809 \mathrm{~kg} / \mathrm{ha}$ ) es igual a $6 \mathrm{ml}$. Esto se debe al método utilizado para analizar los datos del experimento; Cavalheiro (2019), lo realizó considerando las variables independientes (dosis) como cualitativas. Por tanto, luego del análisis de varianza y la aplicación del test de comparación multiple de medias los resultados solo muestran cuál de los tratamientos genera mejor performance (productividad) en el cultivo de soja.

En cambio, en este trabajo, tanto la variable dependiente (productividad) como la variable independiente (dosis) fueron analizados cuantitativamente; siendo lógico, considerando que tanto la productividad como las dosis del enraizante están representadas claramente por números. Los modelos de regresión, mediante predicciones, permiten obtener valores intermedios, esto es, la respuesta del cultivo a la dosis suministrada en cualquier intervalo entre la dosis menor y mayor, asociando la cantidad de enraizante suministrado a la productividad del cultivo.

Analizando la calidad de ajuste del modelo utilizado, se puede afirmar que el modelo linear segmentado con respuesta plato (MLSRP) presenta buen ajuste a los datos analizados. El coeficiente de determinación $\left(\mathrm{R}^{2}\right.$ ) fue 96,70, indicando que el MLSRP consigue explicar el 96,70\% de variación total de los datos. Estos resultados están de acuerdo con Cowles et al. (1995) quienes afirman que la elección de un modelo estadístico se debe basar en criterios dentro de los cuales la lógica biológica del fenómeno 
y el alto valor do coeficiente de determinación $\left(\mathrm{R}^{2}\right)$ no deben estar ausentes. Además de estos criterios, es preciso considerar la maximización de la productividad y del lucro.

Estos resultados refuerzan lo afirmado por Braga (1983) que en estudios con modelos de regresión dosis respuesta concluyo que este tipo de modelos permite detectar la no progresión de la variable de respuesta ante el exceso de determinada dosis (variable independiente).

\section{Conclusión}

En las condiciones en que fue realizado el estudio se concluye que el uso de enraizantes en el cultivo de soja aumenta la productividad. La dosis óptima de enraizante recomendado para el cultivo de soja es $5,08 \mathrm{ml} / \mathrm{kg}$ de semilla. El modelo de regresión lineal con respuesta plato se muestra adecuado para modelar datos que relacionan dosis con productividad una vez que el ajuste del modelo fue bueno.

\section{Referências}

Braga, J. M. (1983). Avaliação da fertilidade do solo: ensaios de campo. Universidade Federal de Viçossa. BR.

Cavalheiro, M. (2019). Efecto de diferentes dosis de enraizante sobre la producción de soja (glycine max, L.) en el departamento de Caaguazú, Tesis. (Ing. Agr.) Asunción, PY: Carrera de Ingenieria Agronómica. FIA. USC.

Cowles, M. K., Best, N., \& Vines, K. (1995). Convergence diagnostics and output analysis. In MRC Bioestatistics Unit Version 0.40 (Vol. 1).

Hermoso, M. (1974). El cultivo de la soja en Argentina. España. Ministerio de Agricultura.

López, O., González, E., Llamas, P. de, Molinas, A., Franco, E., Garcia, S., \& Rios, A. (1995). Mapa de reconocimiento de suelos en la Región Oriental. William \& Heintz Map Corporation.

PNCS. (2016). Plataforma Nacional de Commodities Sustentables.

R Development Core Team. (2020). R: A language and environment for statistical computing. Vienna: R Foundation for Statistical Computing. Disponible en: de http://www.r-project.org.

Veronesi, M. (2014). Evaluación de inoculantes y promotores de crecimiento en un cultivo de soja en Gualeguaychú, provincia de Entre Ríos. Universidad Catolica Argentina.

\section{Histórico do artigo:}

Recebido: 14 de agosto de 2020 . Aprovado: 21 de setembro de 2020. Disponível online: 25 de janeiro de 2021.
Licenciamento: Este artigo é publicado na modalidade Acesso Aberto sob a licença Creative Commons Atribuição 4.0 (CC-BY 4.0), a qual permite uso irrestrito, distribuição, reprodução em qualquer meio, desde que o autor e a fonte sejam devidamente creditados. 